\title{
Zwischen klassischer und moderner Wissenschaftstheorie: Hermann von Helmholtz und Karl R. Popper, erkenntnistheoretisch verglichen ${ }^{1}$
}

\author{
Von GREGOR SCHIEMANN (Darmstadt)
}

Mit seinem Einfluß auf die Entwicklung der Physiologie, Physik und Geometrie ist Hermann von Helmholtz wie kaum ein anderer Wissenschaftler der zweiten Hälfte des 19. Jahrhunderts repräsentativ für die Naturforschung in Deutschland. ${ }^{2}$ Nicht weniger repräsentativ nimmt sich die Entwicklung seiner Wissenschaftsauffassung aus. Während er bis in die späten 60er Jahre einen emphatischen Wahrheitsanspruch der Wissenschaft vertrat, begann er in der nachfolgenden Zeit, die Geltungsbedingungen der wissenschaftlichen Erkenntnis einer Relativierung zu unterwerfen, die zusammenfassend als Hypothetisierung bezeichnet werden kann. Helmholtz' Schriften reflektieren damit die beginnende Erosion einer Wissenschaftsauffassung, deren Anfänge auf Aristoteles zurückgehen und die die neuzeitliche Naturforschung bis ins 19. Jahrhundert hinein bestimmte. Wissenschaftliche Erkenntnis trat seit der Antike mit dem absoluten Anspruch auf Ausschließlichkeit und Unwandelbarkeit auf. In Anlehnung an die Untersuchungen zum Wandel des Wissenschaftsbegriffes im 19. Jahrhundert von Alwin Diemer und Herbert Schnädelbach nenne ich diese Auffassung ,,klassisch“. ${ }^{3}$ Für sie gibt Helmholtz' frühe Position ein typisches Beispiel. Darauf werde ich im dritten Teil meines Beitrages eingehen (3).

Im radikalen Verzicht auf einen emphatischen Wahrheitsanspruch sehe ich das Kennzeichen einer „modernen“ Wissenschaftsauffassung, die in unserer Zeit von Autoren wie Thomas S. Kuhn, Paul K. Feyerabend und Richard Rorty angestrebt wird. ${ }^{4}$ Gewöhnlich wird der moderne wissenschaftstheoretische Wahrheitsverzicht als Reaktion auf die Umbrüche in der Physik, vor allem in der Atomphysik, (spezielle Relativitätstheorie und Quantenmechanik) und in den logischen Grundlagen der Mathematik zu Beginn unseres Jahrhunderts verstanden. ${ }^{5} \mathrm{Da}$ solche Interpretationen zu kurzsichtig sind, läßt sich exemplarisch am Wandel von Helmholtz' Wissenschaftsauffassung vorführen: Im zweiten Teil mei-

1 Mit dem Titel spiele ich auf die Schrift von Joseph Schwertschlager „Kant und Helmholtz, erkenntniss-theoretisch verglichen" (Freiburg 1893) an.

2 Zu Helmholtz' Person vgl. die wissenschaftlich in vielerlei Hinsicht zwar ungenügende, aber an Materialreichtum immer noch unübertroffene Biographie von Koenigsberger (1902 f.) und zu seinem Werk die Aufsatzsammlungen Cahan (Hg.) (1994) - mit ausführlicher Bibliographie - und Krüger (Hg.) (1994). Eine erste Übersicht über Leben und Werk gibt Rechenberg (1994).

3 Diemer (1968), Schnädelbach (1983).

4 Kuhn (1976), Feyerabend (1976), Rorty (1981).

5 Stellvertretend für viele und mit zahlreichen Verweisen: Welsch (1988). 
nes Beitrages werde ich erläutern, wie Helmholtz schon im vergangenen Jahrhundert Ansätze einer Wissenschaftsauffassung entwickelte, die mit seiner eigenen früheren Position unvereinbar waren und in erstaunlichem Umfang in die Richtung der Moderne weisen. ${ }^{6} \mathrm{Al}-$ lerdings verließ er dabei den Horizont der Klassik nicht. Er ist kein Denker des Übergangs von der klassischen zur modernen Wissenschaftsauffassung, sondern ist (in wissenschaftstheoretischer Hinsicht) Klassiker geblieben (2).

Wie nah er der modernen Wissenschaftsauffassung trotzdem bereits gekommen ist, kann ein Vergleich mit Karl R. Poppers Forschungslogik illustrieren. Unter den zeitgenössischen Wissenschaftstheoretikern gehört Popper zu denjenigen, die den revisionsfähigen Charakter der wissenschaftlichen Erkenntnis mit allem Nachdruck und großem Einfluß betont haben. ${ }^{7}$ In seiner Forschungslogik ist die Hypothetisierung der wissenschaftlichen Erkenntnis entschieden weiter vorangeschritten als in Helmholtz' Wissenschaftstheorie. Was sich bei Helmholtz erst vage abzuzeichnen beginnt, ist bei ihm bereits explizit formuliertes Programm geworden. Obwohl Helmholtz und Popper in keiner direkten wissenschaftstheoretischen Entwicklungslinie stehen und Popper sich in seinen Schriften auch nur sehr selten und beiläufig auf Helmholtz bezieht ${ }^{8}$, finden sich dennoch überraschende und bisher nicht beachtete Berührungspunkte, die insbesondere dann hervortreten, wenn man Helmholtz' Wissenschaftsauffassung vor dem Hintergrund von Poppers Forschungslogik betrachtet. Ich werde deshalb mit einer knappen Rekonstruktion von Poppers Position beginnen (1) und sie dann im zweiten Teil zu Helmholtz' später Wissenschaftsauffassung ins Verhältnis setzen.

Vorwegzunehmen ist, daß Popper den absoluten Geltungsanspruch der wissenschaftlichen Erkenntnis allerdings nicht uneingeschränkt verworfen hat. Im Unterschied zu den oben genannten modernen Autoren möchte er - und dies verbindet ihn unmittelbar mit Helmholtz - die klassische Wahrheitsidee als Zielvorstellung der Wissenschaften ausdrücklich aufrechterhalten. Im Vergleich von Popper und Helmholtz werden also nicht nur die Modernität der Helmholtzschen Position, sondern ebenso die durchaus noch klassischen Elemente der Popperschen Forschungslogik deutlich.

(1) Daß alle wissenschaftliche Erkenntnis bloß hypothetischen Charakter habe, ist der zentrale Gedanke von Poppers Wissenschaftstheorie. Popper geht bei der Begründung dieser Ansicht davon aus, daß die Aufgabe der Wissenschaft vor allem theoretischer Art sei

6 Der Wandel von Helmholtz' Wissenschaftsauffassung bzw. Erkenntnistheorie wurde früh bemerkt (vgl. Conrat (1904), Erdmann (1921)). Aber erst in jüngster Zeit wird ihm verstärkte Aufmerksamkeit geschenkt (vgl. König (1968), Hörz und Wollgast (1971), Heimann (1974), Winters (1985), Buchwald (1994), Hatfield (1994), Heidelberger (1994) und Schiemann (1994)). Zu den Invarianten seiner Wissenschaftsauffassung vgl. auch Cahan (1994).

7 Außer Popper und den bereits genannten Wissenschaftstheoretikern Kuhn und Feyerabend ist hier vor allem W.V.O. Quine zu nennen. Poppers Einfluß reicht weit über die Wissenschaftstheorie hinaus. Vgl. Schilpp (Hg.) (1974).

8 Meines Wissens erwähnt Popper Helmholtz nur in: Popper (1930 ff.), XVIII, 30, 207, in: Popper (1956), Bd. 2, 138, Bd. 3, 172. Aus diesen wenigen Bemerkungen kann man entnehmen, daß Popper Helmholtz als einen Vertreter der kantischen Tradition der Physik einordnete (Popper (1956), Bd. 3, 172, Popper (1930 ff.), 30), der ein mechanistisches Reduktionsprogramm vertrat ( Popper (1956), Bd. 2, 138) und mit seiner empiristischen Grundlegung der Geometrie zur Vorgeschichte der Einsteinschen Gravitationstheorie gehört (Popper (1930 ff.), XVIII, 207). 
und in der Erklärung von Phänomenen bestehe. Erklären heißt bei ihm schon das streng logische Geschäft der Deduktion': Aus Prämissen, die mit Gesetzen und Randbedingungen gegeben sind, wird auf Sätze geschlossen, die das zu erklärende Phänomen beschreiben. ${ }^{10}$ Mithin hängt die Wahrheit der Erklärung von der Wahrheit der Prämissen ab.

Über diese kann nun laut Popper aus prinzipiellen Gründen in keinem Fall positiv entschieden werden. Zum einen beruft er sich hierbei auf die logische Unmöglichkeit, Gesetze, die den Charakter von Allaussagen mit unbeschränktem Individuenbereich haben, aus besonderen Beobachtungsaussagen induktiv abzuleiten. Während Gesetze deshalb nicht verifizierbar sind, können sie umgekehrt aber durch Beobachtungsaussagen widerlegt, d.h. falsifiziert werden." (Das Gesetz „Alle Edelmetalle leiten den Strom" wird durch noch so viele singuläre Aussagen über Edelmetalle nicht bewiesen, wohl aber durch die Entdeckung eines einzigen nichtleitenden Edelmetalles strenggenommen ungültig.) Zum anderen ist Popper davon überzeugt, daß sich auch Beobachtungsaussagen nicht verifizieren lassen. Solche Aussagen beziehen sich auf Objekte oder Vorgänge, die unmittelbar wahrnehmbar oder über indirekte Messungen feststellbar sind. Doch weder Wahrnehmungen noch Meßoperationen sind von theoretischen und nur hypothetisch gültigen Vorannahmen frei. Es ist diese „Theoriebeladenheit“" der Erfahrung, die die Geltung der empirischen Basis der Wissenschaft relativiert. ${ }^{12}$ Auf der Ebene der Theorien führt sie rückwirkend zur Einsicht, daß alternative Theorien neue Sichtweisen erschließen können. Damit trägt sie zur Begründung einer pluralen Theorienkonzeption bei. ${ }^{13}$

Wissenschaftliche Erkenntnis ist also bei Popper auf doppelte Weise hypothetisch: Die Wahrheit sowohl der Gesetzes- als auch der Beobachtungsaussagen kann nur vermutet werden. ${ }^{14}$ Modern ist seine Wissenschaftstheorie freilich nicht aufgrund dieser Charakterisierung, sondern aufgrund ihrer positiven Bewertung für die Forschungsmethodologie. „Mit dem Idol der Sicherheit, auch der graduellen“, sagt Popper, , fällt eines der schwersten Hemmnisse auf dem Weg der Forschung ". ${ }^{15}$ Erst die Beseitigung absoluter Geltungsansprüche setzt Kreativität und Rationalität frei. Mit dem Zwang, Theorien induktiv zu rechtfertigen, verabschiedet Popper alle wissenschaftlichen Begründungspflichten ebenso wie die logischen Regeln für die Aufstellung von Theorien. Neue Theorien sieht er aus einem Prozeß von Versuch und Irrtum hervorgehen, in dem das Glück nicht weniger zum Fortschritt beiträgt als der schöpferische Einfall. ${ }^{16}$

Statt ihre Wissenschaftlichkeit an der Möglichkeit der Bestätigung von Voraussagen zu messen, macht Popper die Falsifizierbarkeit zur charakteristischen Eigenschaft einer wis-

9 Wie später Hempel und Oppenheim (1948). Mit Popper beziehe ich den Begriff der Deduktion bzw. der ,deduktiven Überprüfung von Theorien“ nicht nur auf die empirische Prüfung (mit dem Ergebnis der Verifikation oder Falsifikation einzelner abgeleiteter Sätze), sondern auch auf die Prüfung der Widerspruchsfreiheit und des nicht tautologischen Charakters von Theorien sowie auf intertheoretische Vergleiche (Popper (1935), 7 f.).

10 Popper (1935), $31 \mathrm{ff}$.

11 Ebd., 3 ff.

12 Ebd., $58 \mathrm{ff}$.

13 Schäfer (1988), $58 \mathrm{f}$.

$14 \mathrm{Vgl}$. die Definition des Hypothesenbegriffes in Popper (1973), 21.

15 Popper (1935), 225.

16 Ebd., 6 f., Popper (1963), 49 ff., Popper (1973), 168. 
senschaftlichen Theorie. Danach muß eine empirische Theorie, um wissenschaftlich zu heißen, in logisch einwandfreier Weise angeben, welche Vorgänge in ihrem Gegenstandsbereich nicht vorkommen dürfen. ${ }^{17}$ Sie enthält damit die provokative Aufforderung an ihre potentiellen Kritikerinnen und Kritiker, genau diese Vorgänge zu suchen, um durch ihren Nachweis zur Widerlegung der Theorie beizutragen. Je größer die Klasse der ausgeschlossenen Vorgänge, desto größer ist nach Popper der ,empirische Gehalt" einer Theorie. Und je mehr Widerlegungsversuche scheitern, desto „,bewährter“ ist eine Theorie. Paradox formuliert erweist sich der Erfahrungsbezug im erfolgreich durchgesetzten Erfahrungsverbot. In dieser seltsam anmutenden Bestimmung reflektiert sich die wohlbekannte hoch entwickelte Spezialisierung moderner Wissenschaft. Deren enge Gegenstandsbereiche sind um so besser bestimmt, je schärfer sie sich gegen andere abgrenzen lassen.

Obwohl Poppers Methodologie der Theorienvielfalt in vielerlei Hinsicht das Wort redet ${ }^{18}$, hält er doch daran fest, daß die Wissenschaft nach einer umfassenden wahren Theorie streben solle. Grundlage dieser Inkonsistenz sind Poppers korrespondenztheoretischer Wahrheitsbegriff und sein metaphysischer Realismus. Metaphysisch, und d.h. bei Popper auch hypothetisch, nennt er diesen Realismus, weil er weder nachweisbar noch widerlegbar sei und sich darin nicht von der idealistischen Gegenposition unterscheide. Wie andere metaphysische Annahmen, sei der Realismus von großer heuristischer Nützlichkeit. Gestützt auf das Zeugnis des Alltagsverstandes postuliere er eine hinter den Erscheinungen liegende Wirklichkeit. Diese eine Wirklichkeit in immer zutreffenderer Weise zu erfassen, müsse das Ziel der Wissenschaft sein. Ihre Theorien mißt Popper deshalb auch daran, inwieweit sie mit der Wirklichkeit übereinstimmen, d. h. inwieweit sie wahr sind und - für ihn gleichbedeutend - sich fortschreitend der Wahrheit im Sinne einer vollständigen und allein gültigen Erklärung der Welt annähern. ${ }^{19}$

(2) Die von Popper übernommene klassische Wahrheitsidee bezeichnet ein unerreichbar weit entferntes Ziel. Sie ist - mit Kant zu sprechen - regulative Idee der Forschung. ${ }^{20}$ In ähnlicher Weise ist auch noch die Fortschrittsorientierung des späten Helmholtz der klassischen Wissenschaftsauffassung verpflichtet. In seiner berühmten Rede „Die Thatsachen in der Wahrnehmung“ von 1878 bezeichnet er die Vorstellung, daß „das Begreifen (der Naturerscheinungen) zu vollenden sein“" werde, als „das regulative Princip unseres Denkens“. ${ }^{21}$ Wie Popper verbindet Helmholtz die Idee einer absolut gültigen Naturerklärung mit einer realistischen Auffassung der Außenwelt. Mit Berufung auf die - so wörtlich - ,tägliche (...) Wahrnehmung " nimmt er eine bewußtseinsunabhängige Wirklichkeit an, stellt diesen Realismus explizit dem Idealismus als unwiderlegbare und deshalb „metaphysische Hypothese“ zur Seite und spricht beiden ,vollkommene wissenschaftliche Berechtigung“ zu. ${ }^{22}$

17 Popper (1935), 43 ff.

18 Popper (1973), 25 ff., 65 ff. u.ö.

19 Popper (1973), 49 ff., 347 ff., Popper (1963), 215 ff.

20 Kant (1787), $702 \mathrm{ff}$.

21 Helmholtz (1878a), 243. Vgl. Popper (1973), 42. Während Helmholtz die Begreifbarkeit der Natur an die Annahme eines ,letzte(n) Unveränderliche(n) als Ursache der beobachteten Veränderungen“" knüpft (a.a.O.), lehnt Popper einen derartigen „Essentialismus" ab (Popper (1973), 217 ff.).

22 Helmholtz (1878a), 239. 
Helmholtz teilt mit Popper ferner den empiristischen Standpunkt in der Begründung wissenschaftlicher Geltungsansprüche. Allerdings vertritt er dabei kein ausschließlich deduktivistisches, sondern ein wesentlich induktivistisches Konzept. Die Hypothetizität des Geltungsanspruches von Gesetzesaussagen entspringt bei ihm der - auch von Popper formulierten - Einsicht in die Unabschließbarkeit von Induktionen. Unter Induktion versteht Helmholtz in wissenschaftlicher Hinsicht eine Methode, mit der von besonderen Erfahrungen auf allgemeine Gesetzesaussagen geschlossen wird. Sie ist für die Entdeckung und Rechtfertigung von Gesetzen leitend und bezeichnet deren empirischen Ursprung. ${ }^{23}$ Unabschließbar ist für ihn die induktive Methode allein schon deshalb, weil sie nur auf der Beobachtung von Einzelfällen basiert und auf dieser Grundlage nicht ausgeschlossen werden kann, daß Erfahrungen in der Beobachtung vorkommen, die einem Gesetz oder der Naturgesetzlichkeit überhaupt widersprechen. ${ }^{24}$ „Alle Kenntniss der Naturgesetze“, schreibt er zwei Jahre vor seinem Tod, ,,ist inductiv, keine Induction ist je absolut fertig. “25

Für Helmholtz' späte Wissenschaftstheorie ist kennzeichnend, daß er die Unabschließbarkeit von Induktionen weitergehend mit seiner empiristischen Wahrnehmungstheorie begründet. Induktionsschlüsse sind seiner Auffassung nach auch für elementare Prozesse konstitutiv, die die alltägliche Wahrnehmung bestimmen und aller Erkenntnis zugrunde liegen. Ihre Irrtumsanfälligkeit und Wandelbarkeit könne im Prinzip weder von der Naturwissenschaft noch von der Logik verhindert werden. ${ }^{26}$ Während Popper die Geltung der klassischen Logik in seiner deduktiven Wissenschaftstheorie nicht bezweifelt, gerät sie bei Helmholtz, wenn auch erst ansatzweise, bereits in den Sog der Hypothetizität.

Das Ergebnis einer naturwissenschaftlichen Induktion bezeichnet Helmholtz als Hypothese. Ihre Wahrheitsoffenheit kann in einem weiteren Schritt der Verifikation, den Helmholtz auch „Deduktion“ nennt, sukzessiv reduziert werden. Deduktion ist bei ihm eine Methode zur empirischen Bestätigung von Gesetzeshypothesen und unterscheidet sich in ihrer logischen Form nicht von der der Induktion. ${ }^{27}$ „Glauben wir ein Gesetz gefunden zu haben, dann tritt ... das Geschäft des Deducirens ein. Dann haben wir die Consequenzen unseres Gesetzes möglichst vollständig abzuleiten, aber freilich zunächst nur, um sie an der Erfahrung zu prüfen, so weit sie sich irgend prüfen lassen, und um durch diese Prüfung zu entscheiden, ob das Gesetz sich als gültig bewähre und in welchem Umfange. "28

23 Helmholtz (1862), 169 ff., Helmholtz (1856 ff.), 447 ff., Helmholtz (1874a), 414 ff., Helmholtz (1892), 338 ff. u.ö. Helmholtz' Verständnis der Induktion deckt sich im wesentlichen mit dem - auch von Popper geteilten - heute noch üblichen, nach dem man mit einem ,induktiven Schluß oder Induktionsschluß ... einen Schluß von besonderen Sätzen, die z. B. Beobachtungen, Experimente usw. beschreiben, auf allgemeine Sätze, auf Hypothesen oder Theorien" bezeichnet (Popper (1935),3).

24 Helmholtz (1856 ff.), 447 f., 451 f., Helmholtz (1885 ff.), 581 f.

25 Helmholtz (1892), 358.

26 Helmholtz (1878a), 233, 243, Helmholtz (1892), 359 f., Helmholtz (1885 ff.), 576 ff.

$27 \mathrm{Vgl}$. Anm. 23. Zur logischen Äquivalenz von Induktion und Deduktion vgl. Mill (1843), 1. Teil, $213 \mathrm{f}$. Helmholtz thematisiert das formale Verhältnis von Induktion und Deduktion nicht. In seiner Bestimmung der Induktion beruft er sich aber ausdrücklich auf Mill (Helmholtz (1856 ff.), $447 \mathrm{f}$.), dessen Wissenschaftstheorie für ihn vermutlich von großer Bedeutung war.

28 Helmholtz (1878c), 183. 
Insofern das „Geschäft des Deducirens“ eine hinreichend logische Struktur des theoretischen Kontextes, in dem das Gesetz formuliert ist, voraussetzt, besteht eine - wenn auch nur entfernte - Verwandtschaft zu Poppers deduktiver Forschungslogik. Im Gegensatz zu dieser denkt Helmholtz aber nicht an die Falsifikation der negativen Konsequenzen bzw. Voraussagen, sondern allein an die Verifikation der positiven. Folglich kann bei ihm die Überprüfung durch Deduktion sowenig wie die Induktion abschließbar sein. Wenn Helmholtz davon spricht, man habe zu prüfen, ,in welchem Umfange“ sich ein Gesetz ,als gültig bewähre“, bringt er zum Ausdruck, daß die Gesetzesgeltung von der Eingrenzung des Anwendungsbereiches abhängig ist. Wie Popper trägt er damit dem hohen Maß wissenschaftlicher Spezialisierung Rechnung. Bei Helmholtz führt die Umfangsbestimmung aber nicht zur Begründung der Hypothetizität, sondern umgekehrt zu ihrer Minimierung. Vermittels der Deduktion werde nämlich „das hypothetische Element um so mehr heraus geschafft ..., je weiter die betreffende Untersuchung ins Specielle durchgeführt" werden könne. ${ }^{29}$ Dies sei jedoch eine Arbeit, die eigentlich nie aufhöre. ${ }^{30}$

Bis zu welchem Grad sich diese Hypothesenbereinigung vorantreiben läßt, hängt wesentlich vom Geltungscharakter der empirischen Basis ab, der bei Popper (wegen der „Theoriebeladenheit") nur relativ ist. Bei Helmholtz enthält die Zurückführung der wissenschaftlichen Geltungsbedingungen auf elementare Wahrnehmungserlebnisse, die sowohl durch alltägliche Erfahrungen als auch durch theoretische (beispielsweise geometrische) Vorannahmen geprägt sind, eine erste Relativierung der empirischen Basis. Außerdem lassen sich verschiedene Aspekte seiner Erkenntnis- und Wissenschaftstheorie angeben, die im Rückblick als Vorformen der Popperschen „Theoriebeladenheit“ angesehen werden können. So ist seinen Bemerkungen zum hypothetischen Charakter von Realismus und Idealismus zu entnehmen, daß er empirische Begriffe, ohne die Beobachtungsaussagen nicht vorgenommen werden können, an erkenntnistheoretische Voraussetzungen gebunden sieht. ${ }^{31}$ Welche Bedeutung wissenschaftliche Theorien für die Repräsentation der empirischen Basis haben können, zeigt sich in seinen berühmten Arbeiten zur empirischen Begründung der nichteuklidischen Geometrien. In diesen führt er anschaulich vor, daß Aussagen über den physikalischen Raum nicht mehr eindeutig, sondern nur in verschiedener, aber äquivalenter Weise möglich sind.

Im Anschluß an die Arbeiten von Bernhard Riemann leitet Helmholtz aus den Eigenschaften der formunveränderlichen und freien Bewegung von Festkörpern her, daß außer der euklidischen Geometrie auch nichteuklidische geometrische Systeme möglich sind. Welches System im Raum realisiert sei, könne man durch Messungen empirisch ermitteln. Für die physikalischen Rechnungen sei aber die reale Struktur des Raumes unerheblich, insofern sich die verschiedenen Systeme äquivalent ineinander überführen ließen. ${ }^{32}$

29 Helmholtz (1897 ff.), Bd. I.1, 19.

30 Helmholtz (1878c), 183.

31 Helmholtz (1878b), $648 \mathrm{f}$.

32 Riemann (1867), Helmholtz (1868a), Helmholtz (1868b), Helmholtz (1878b) und die eher populären Darstellungen Helmholtz' (1870), 1 ff., Helmholtz' (1878a), 229 ff. Ich beschränke mich hier auf die Erwähnung nur derjenigen Elemente in Helmholtz' geometrischen Arbeiten, die rückblickend einem modernen Verständnis wissenschaftlicher Erkenntnis verwandt sind. Helmholtz 
Somit hängen die Darstellung des Raumes und die darauf bezogenen physikalischen Gesetze von der Entscheidung über das jeweils verwendete geometrische System ab. Gab es früher hierfür nur das euklidische System, dessen Struktur mit der Struktur des Raumes identifiziert wurde, so sind jetzt Aussagen über das empirisch im Raum Gegebene nur noch unter der Voraussetzung von theoretischen und im Prinzip frei wählbaren Annahmen möglich und deshalb nur relativ gültig. Mit dieser Einsicht nähert sich Helmholtz einer konventionalistischen Auffassung der Geometrie, wie sie später von Henri Poincaré begründet wird. Mit der Vielfalt der geometrischen Darstellungsmöglichkeiten gibt er ein frühes Beispiel für einen Theorienpluralismus, der (wie bei Popper) mit dem Verlust eines unzweifelhaft Gegebenen einhergeht.

Was ich bisher über Helmholtz' späte Wissenschaftsauffassung gesagt habe, läßt sich dahingehend zusammenfassen, daß sich schon bei Helmholtz eine doppelte, die Gesetze und die empirische Basis betreffende Hypothetizität der wissenschaftlichen Erkenntnis findet. ${ }^{33}$ Die Relativierung des Geltungsanspruches wird nicht nur als vorübergehende Wahrheitsoffenheit begriffen. Im Hinblick auf die empirische Basis hat sie prinzipiellen Charakter, der auf die Ebene der Gesetze zurückwirken muß. Es kann deshalb keine vollständig hypothesenfreie Gesetzeserkenntnis mehr geben. Helmholtz spricht dies als Einsicht aus, kein Gesetz sei vor Abänderungen sicher. Der „echte Naturforscher“, schreibt er 1877, überlege sich „,bei jeder neuen fremdartigen Erscheinung, ob nicht die bestbewährten Wirkungsgesetze längst bekannter Kräfte eine Abänderung erhalten“ müßten; natürlich könne „,es sich dabei nur um eine Abänderung handeln, die dem ganzen Schatze der bisher aufgesammelten Erfahrungen nicht" widerspreche. ${ }^{34}$ Auch wenn es sich hier nur um graduelle Abänderungen handeln würde, so steht doch bereits fest, daß selbst die „bestbewährten“ Gesetze davon betroffen sein können. ${ }^{35}$

Weil der ursprünglich hypothetische Charakter von Gesetzen nicht restlos zu beseitigen ist $^{36}$, muß die Wissenschaft für Revisionen offenbleiben. Helmholtz formuliert diese For-

selbst hat mit seinen Arbeiten einen durchaus klassischen Zweck verfolgt. Er wollte der von Riemann vertretenen hypothetischen Auffassung der Geometrie eine auf unzweifelbare „Thatsachen“ gegründete entgegensetzen. Dementsprechend kann der Titel von Helmholtz (1868b) („Ueber die Thatsachen, die der Geometrie zum Grunde liegen“) als direkte Antithese zu Riemann (1867) („Ueber die Hypothesen, welche der Geometrie zugrunde liegen“) gelesen werden.

33 Von wissenschaftlichen Hypothesen kann bei Helmholtz in dreifachem Sinn die Rede sein: im Sinn von noch nicht gefundenen oder fik tiv angenommenen unsichtbaren Entitäten, von nicht hinreichend verifizierten oder nicht vollständig verifizierbaren Gesetzen und schließlich im Sinn von unwiderlegbaren erkenntnistheoretischen Voraussetzungen. Vgl. Schiemann (1994).

34 Helmholtz (1878c), 183.

35 Helmholtz hat nicht nur spezielle Naturgesetze, sondern auch die allgemeinen Prinzipien der mathematischen Naturforschung - zu denken wäre hier z.B. an die Lagrange-Gleichung der Mechanik - für fallibel gehalten: ,Was die ... (naturwissenschaftlichen Axiome) betrifft, so sind sie theils von bestrittener Richtigkeit, theils einfache Folgerungen aus dem Princip der Causalität, das heisst aus dem Triebe unseres Verstandes, alles Geschehende als gesetzmässig, ... zu betrachten." (Helmholtz (1878b), 642). Zur hypothetischen Auffassung der Kausalität, wie sie der späte Helmholtz vertrat: Helmholtz (1878a), 243 f., Helmholtz (1885 ff.), 593, und das in Koenigsberger (1902 f.), Bd. I, 247, wiedergegebene Nachlaßfragment.

36 ,Unwürdig eines wissenschaftlich sein wollenden Denkers aber ist es, wenn er den hypothetischen Ursprung seiner Sätze vergisst." Helmholtz (1878a), 239. 
derung auch implizit, wenn er sich gegen „dogmatische Systeme“ wendet, denen der wissenschaftliche Status abgehe. Bei diesen Gelegenheiten findet die Nähe zu Poppers Forschungslogik einen vielleicht nur zufälligen, nichtsdestoweniger aber bemerkenswerten A usdruck. Wie später Popper wählt Helmholtz als Beispiel für den Dogmatismus auch die Astrologie, um sie der Astronomie als einer Wissenschaft gegenüberzustellen. ${ }^{37}$ Als Charakteristikum der Unwissenschaftlichkeit führt er wörtlich die „Infallibilität“ des Dogmatismus an: „Charakteristisch aber für die Schulen, die auf solchen als Dogmen angenommenen Hypothesen ihr System errichteten, ist die Intoleranz ... Die überzeugten Anhänger müssen deshalb für jeden einzelnen Theil eines solchen Gebäudes denselben Grad von Infallibilität in Anspruch nehmen ..." “38

Die Einsicht in die Hypothetizität der wissenschaftlichen Erkenntnis hat faktisch für Helmholtz durchaus forschungsfördernde Wirkung. Seine Wissenschaftsauffassung wird gegenüber alternativen Konzeptionen toleranter ${ }^{39}$, läßt in einem Gegenstandsbereich verschiedene Theorien $\mathrm{zu}^{40}$, löst sich von ontologisch begründeten absoluten Geltungsansprüchen und lockert die inhaltlichen Bestimmungen der wissenschaftlichen Zielsetzung. ${ }^{41}$ Modern wäre seine Wissenschaftsauffassung aber erst zu nennen, wenn er die Hypothetizität zur ersten Tugend der Wissenschaftlichkeit erheben würde. Davon ist er jedoch weit entfernt. Für ihn bleiben Hypothesen in der Wissenschaft ein Makel, den es besser nicht gäbe, und der, wenn er sich denn schon nicht restlos beseitigen läßt, doch jedenfalls minimiert werden müsse. Zudem ist Helmholtz' späte Wissenschaftsauffassung nicht frei von widersprüchlichen Äußerungen. Die Begründung der Hypothetizität steht mitunter noch völlig unvermittelt neben beibehaltenen Elementen seiner früheren klassischen Auffassung.

So unterscheidet er terminologisch strikt zwischen induktiv aufgestellten Hypothesen und erfolgreich bestätigten Gesetzen, die von ihm - ungeachtet ihrer ,nur approximativen Erweisbarkeit" 42 - zeitlebens mit dem Anspruch auf strenge Allgemeingültigkeit versehen werden. ${ }^{43}$ Helmholtz verfolgt überdies auch eine Strategie zur Ausschaltung des auf Induktionen zurückgehenden hypothetischen Gehaltes. Es handelt sich um ein nichtinduktives Verfahren, das trotz seiner äußerlichen Ähnlichkeit mit Poppers Forschungsmethodologie mit dieser nur verwechselt werden könnte, wenn man die Differenz zwischen Helmholtz' induktiv ausgerichtetem und Poppers deduktivem Ansatz vergäße. Vermutlich mit

37 Helmholtz (1878c), 188, Helmholtz (1874b), 433, Popper (1963), 37 f., 188 u.ö.

38 Helmholtz (1878c), $175 \mathrm{f}$.

$39 \mathrm{Vgl}$. seine zweite Rede zu Goethe: Helmholtz (1892).

40 Neben der Geometrie gilt dies auch - allerdings nur im Sinn einer vorübergehenden Zulässigkeit - für die Elektrodynamik (zu dieser vgl. Kaiser (1994) und Buchwald (1994)) und für die Wahrnehmungstheorie (vgl. Turner (1994)).

41 Dies läßt sich an der Entwicklung seiner mechanistischen Naturauffassung nachweisen (vgl. Schiemann (1994)).

42 Helmholtz (1878a), 393.

43 Helmholtz (1854), 82, Helmholtz (1862), 169 f., Helmholtz (1869), 347 f., Helmholtz (1878a), 240, Helmholtz (1886), 318, Helmholtz (1887), 283, 287, Helmholtz (1892), 339, 353 f. In Poppers nichtinduktiver Forschungslogik ist die Forderung nach strenger Allgemeingültigkeit, die im Gesetzesbegriff immer schon impliziert ist und von ihm für die Forschungspraxis auch ausdrücklich erhoben wird (z.B. Popper (1973), 220), natürlich unproblematisch. 
Blick auf eigene Erfahrungen glaubt Helmholtz in seiner späten Wissenschaftsauffassung, daß neben der Induktion auch die Wirksamkeit einer intuitiven Gabe zur Aufstellung von neuen Gesetzen führen könne. Im Gegensatz zu den induktiv gefundenen Gesetzen wie im Gegensatz zur logisch nicht regelbaren Entdeckung von Gesetzen bei Popper nennt Helmholtz das Ergebnis einer intuitiven Erkenntnis jedoch nicht hypothetisch, sondern rückt es in die Nähe religiöser Erleuchtungserlebnisse. Die wissenschaftliche Intuition sei „durch einen tiefen Blick in den Zusammenhang des Ganzen erzeugt“"44, eine „plötzlich auftauchende Einsicht, (eine) ... Divination, ... eine Art göttlicher Eingebung“" ${ }^{45}$

(3) Die Verklärung des wissenschaftlichen Erkenntnisanspruches, wie sie in solchen Bemerkungen zum Ausdruck kommt, ist bemerkenswerterweise kein Relikt aus Helmholtz' früher Wissenschaftsauffassung. Bis zum Ende etwa der 60er Jahre grenzt er sich strikt gegen alle nichtinduktiv gewonnene Erkenntnis ab. ${ }^{46}$ Zudem zeichnet er in dieser Zeit die Induktion als naturwissenschaftliches Verfahren gegenüber der geisteswissenschaftlichen Methodik mit einem besonderen Geltungsanspruch aus. Die naturwissenschaftliche Induktion habe den Charakter einer "logischen Induktion“, weil sie von den „Einzelfälle(n) der Beobachtung und Erfahrung“ „bis zur vollendeten Form des logischen Schließens, ... zur Aufstellung ausnahmslos geltender Gesetze durchgeführt werden" könne. ${ }^{47}$

Sowenig Helmholtz zu dieser Zeit an der uneingeschränkten Geltung logischer oder mathematischer Sätze zweifelt ${ }^{48}$, sowenig zweifelt er an der absoluten Sicherheit des induktiven Verfahrens. Dementsprechend nennt er das Ergebnis von Induktionen nicht ,hypothetisch“ (er verwendet den Ausdruck Hypothese anfänglich überhaupt nur ausnahmsweise) und sieht keine Notwendigkeit, es um eine nachgeordnete Deduktion zu ergänzen. Die Gültigkeit von induktiv ermittelten Gesetzen sei lediglich noch ,an verwickelteren Fällen“" zu prüfen. ${ }^{49}$

Seine frühe, noch ausnahmslos induktiv verfaßte Wissenschaftsauffassung stützt Helmholtz auf die besonderen Merkmale des experimentellen Verfahrens. In Experimenten könne man ,willkürlich die Bedingungen verändern ..., unter denen der Erfolg“ eintritt, und „sich deshalb auf eine nur kleine Zahl charakteristischer Fälle der Beobachtung beschränken ..., um das Gesetz zu finden“. ${ }^{50}$ Die unendliche Zahl von Fällen, die mit einem allgemein gültigen Gesetz erfaßt wird, reduziert sich in der technischen Praxis von Versuchen auf die Variation einer einzigen Bedingung unter sonst gleichbleibenden, fixierten Umständen. Dem Naturgesetz entspricht ein einziger charakteristischer Versuchsaufbau. Dieser idealisierenden Darstellung der experimentellen Arbeit liegt beim Realisten Helmholtz natürlich nicht die Vorstellung zugrunde, daß die Gesetze wie die künstlich konstruierten Versuchsapparaturen Erfindungen des menschlichen Geistes seien. Gesetze werden seinem Empirismus gemäß in der Praxis der Forschung nicht erzeugt, sondern „gefunden“.

Helmholtz' frühe Wissenschaftsauffassung steht in der Tradition empiristischer Konzep-

44 Helmholtz (1878c), 185.

45 Helmholtz (1892), 348 (Hervorheb. im Text). Vgl. Helmholtz (1878a), 232 f., Helmholtz (1881), 253, 257.

46 Vor allem in seiner Polemik gegen Goethe und die romantische Naturphilosophie, vgl. Helmholtz (1853), 40 ff., Helmholtz (1855), 89.

47 Helmholtz (1862), S. 175, 171.

48 Ebd., 175 f.

49 Ebd., 177.

50 Ebd. 
tionen, wie sie in der Naturforschung zu Beginn der Neuzeit paradigmatisch von Galileo Galilei und Isaac Newton vertreten wurden - eine Tradition, die sich erkenntnistheoretisch kaum schon mit einer modernen Forschungslogik vergleichen läßt. Ganz im Bann des frühneuzeitlichen Wahrheitspathos befangen, ging beispielsweise Galilei davon aus, im Experiment würden abstrakte und konkrete Aussagen wechselseitig aufeinander abgestimmt und zur Übereinstimmung gebracht. Die logische Struktur der so erhaltenen Gesetze zeuge von einer Wesensbestimmung der Natur, die rein nur in technischen Konstrukten vorliege. ${ }^{51}$

Im Gegensatz zur späteren wahrnehmungstheoretisch begründeten Hypothetisierung der wissenschaftlichen Erkenntnis stellt der frühe Helmholtz die experimentell gefundene objektive Wahrheit dem bloß subjektiven Zeugnis der Wahrnehmung gegenüber. Seine Wissenschaftsauffassung ist noch nicht reflexiv geworden. Naturwissenschaft, insbesondere die von ihm vertretene Physiologie, hat die Wahrnehmung zum Gegenstand, ohne selbst Gegenstand der Wahrnehmung zu sein. Daß subjektive Wahrnehmungserlebnisse bloß relative Geltung haben, wird von der Physiologie mit absolutem Geltungsanspruch festgestellt. Später erst heißt es umgekehrt, auch wissenschaftliche Experimente können die bloß relative Geltung einer - modern ausgedrückt - lebensweltlich fundierten Wahrnehmung im Prinzip nicht transzendieren. ${ }^{52}$

Dem absoluten Geltungsanspruch entspricht eine Fixierung der wissenschaftlichen Zielsetzung auf bestimmte Inhalte. Statt der bloß regulativen Orientierung am abstrakten Postulat der Begreifbarkeit der Natur gibt Helmholtz auf kaum mißverständliche Weise zu verstehen, daßer an die Möglichkeit einer tatsächlich durchgeführten vollständigen und allein gültigen Naturerklärung glaubt. ${ }^{53}$ Eine derart wahre Erklärung zu erreichen, war das eigentliche Motiv der klassischen Wissenschaftsauffassung, wie sie von der neuzeitlichen Naturforschung begründet wurde. ${ }^{54}$ Mit dieser teilt Helmholtz (zeitlebens) die Orientierung am Paradigma der Mechanik. 1869 betont er ein letztes Mal, es sei das „Endziel der Naturwissenschaften“, ,sich in Mechanik aufzulösen“.55

Obwohl sich in Helmholtz' Wissenschaftsauffassung bereits in den 50er und 60er Jahren Veränderungen abzeichnen, verbleibt sie noch streng im Rahmen der klassischen Konzeption. Für die danach einsetzende Erosion der bisherigen Wahrheitsgewißheiten findet man in diesem Zeitraum erstaunlich wenige und kaum eindeutige Anhaltspunkte. ${ }^{56}$ Überdies ist

51 Vgl. Galilei (1632), 219 f., Galilei (1638), 5, Galilei (1890 ff.), Bd. VI, 232, Bd. VII, 211.

52 Dieser Wandel läßt sich durch einen Vergleich von Passagen, die Helmholtz in der 2. Auflage des „Handbuches der Physiologischen Optik“ gestrichen hat (insb. Helmholtz (1856 ff.), 441 ff.), mit den in dieser Auflage neu aufgenommenen (insb. Helmholtz (1885 ff.), $590 \mathrm{ff}$.) verdeutlichen.

53 Helmholtz (1847), 4 ff., Helmholtz (1862), 182, Helmholtz (1869), 377 ff.

54 Sofern die Erreichbarkeit der vollständigen Wahrheit über die Natur nicht ausdrücklich, wie beispielsweise bei Francis Bacon und René Descartes, behauptet wurde, war die noch zu erwartende Entwicklung des Naturwissens nicht als endloser Prozeß konzipiert (so bei Galileo Galilei, Johannes Kepler und Isaac Newton).

55 Helmholtz (1869), 379.

56 Erste Anhaltspunkte für den verstärkten Einfluß der Wahrnehmungstheorie auf die Wissenschaftsauffassung findet man im $\S 26$ von Helmholtz (1856 ff.). Elemente einer modernen Wissenschaftsauffassung sind auch - wie gezeigt - in Helmholtz' Arbeiten zur Geometrie aus den Jahren 1868 bis 1870 impliziert (vgl. Anm. 32). Physikimmanente Triebkräfte für die Entwicklung von Helmholtz' Wissenschaftsauffassung kann man Winters (1985) entnehmen. 
auffällig, daß sich der Wandel in einem doch sehr kurzen Zeitraum zwischen dem Ende der $60 \mathrm{er}$ und dem Anfang der 70er Jahre vollzieht. Bereits 1874 lassen sich die Konturen von Helmholtz' neuer Position rekonstruieren. ${ }^{57}$

Man kann fragen, welche äußeren Faktoren zum Wandel beigetragen haben mögen. Von großer Wichtigkeit ist hierbei sicher Helmholtz' Rezeption des englischen Empirismus und der Wissenschaftsauffassungen englischer Physiker. ${ }^{58}$ Der Wandlungsproze $\beta$ fällt ferner beruflich mit seiner stärkeren Hinwendung zu physikalischen Fragestellungen zusammen. ${ }^{59}$ Nicht auszuschließen ist, daß die Transformation seiner Wissenschaftsauffassung durch die Krise seiner mechanistischen Naturauffassung maßgeblich angeregt wurde, deren begrenzte Reichweite - von Helmholtz nicht unbemerkt - in den 70er Jahren zutage trat. ${ }^{60}$ Zum einen sah sich die von ihm angenommene atomistische Ontologie wachsender Kritik ausgesetzt. Während Helmholtz noch am Ende der 60er Jahre überzeugt war, die antike Elementenlehre habe durch chemische Forschungen endlich und endgültig eine wissenschaftliche Bestätigung gefunden, mußte er schon Ende der 70er Jahre auf ,neue Zerlegungen der jetzt bekannten chemischen Elemente" gefaßt sein. ${ }^{61}$ Zum anderen kamen Zweifel daran auf, ob die elementaren Kräfte auf atomarer Ebene in ihrer Struktur mit der Vorstellung instantan wirkender Zentralkräfte, die Helmholtz seiner Naturauffassung zugrunde gelegt hatte, übereinstimmten. Hier waren es vor allem die elektrodynamischen Erscheinungen und ihre mathematische Darstellung durch James Clerk Maxwell, die das alte mechanistische Weltbild im vergangenen Jahrhundert erschütterten. ${ }^{62}$

57 Helmholtz (1874a) und Helmholtz (1874b). In den meisten Untersuchungen zum Wandel von Helmholtz' Wissenschaftsauffassung (vgl. Anm. 6) wird ein Entwicklungseinschnitt behauptet. Je nachdem, welchen Elementen des Wandels vor allem Beachtung geschenkt wird, fällt seine Datierung unterschiedlich aus. Insgesamt liegen die bisher festgestellten Einschnitte im Zeitraum zwischen 1862 (Hatfield (1994)) und 1871 (Buchwald (1994) und Schiemann (1994)).

58 Nur die wichtigsten Hinweise können hier gegeben werden: Von seinen seit 1853 unternommenen Reisen nach England berichtet Koenigsberger (1902 f.); 1870 gibt Helmholtz ein Buch von John Tyndall über Michael Faraday heraus; 1871 und 1874 den 1. und 2. Teil des 1. Bandes des „Handbuches der theoretischen Physik" von William Thomson und Peter Guthrie Tait; zu James Clerk Maxwell vgl. insb. Helmholtz (1881), 249 ff., und Helmholtz (1882 ff.), Bd. 3, 209. Auf den (- allerdings nicht in Richtung einer Hypothetisierung weisenden -) Einfluß englischer Physiker hat insbesondere Heidelberger (1994) hingewiesen. Zur Rezeption des englischen Empirismus vgl. Anm. 27.

591871 beendet Helmholtz seine Tätigkeit als Professor für Physiologie in Heidelberg und wird Professor für Physik in Berlin.

60 Exemplarisch für die Kritik am mechanistischen Erklärungsprogramm sind Mach (1872) und Kirchhoff (1876).

61 Helmholtz (1878a), 240, und demgegenüber z.B.: Helmholtz (1869), $378 \mathrm{f}$.

$62 \mathrm{Da} 3$ schon Michael Faradays geometrische Theorie der magnetischen Kraftlinien, an der die Maxwellsche Elektrodynamik unmittelbar anknüpft, grundsätzlich in „Gegensatz“ zu allen physikalischen Theorien steht, ,welche mit Atomen und in die Ferne wirkenden Kräften operiren“, bemerkt Helmholtz bereits 1871 (Helmholtz (1884), Bd. 2, 47). Erste eigene Zweifel an der Struktur grundlegender Kräfte finden sich in den 1881 verfaßten Zusätzen zu Helmholtz (1847) und in Helmholtz (1882 ff.), Bd. 2, $1003 \mathrm{ff}$. 
(4) Die Erschütterung ehemals uneingeschränkt geltender Erkenntnisse kennzeichnet auch den historischen Kontext, in dem Poppers Forschungslogik entstanden ist. Seine Wissenschaftstheorie gehört zu den intellektuellen Reaktionen auf die eingangs erwähnten fundamentalen Wandlungen in der Physik und der Mathematik, die sich in den ersten beiden Jahrzehnten unseres Jahrhunderts vollzogen und die in Poppers Werk zeitlebens zentrale Bezugspunkte geblieben sind. ${ }^{63}$ Mit der von Helmholtz und Popper formulierten hypothetischen Auffassung der wissenschaftlichen Erkenntnis wird auf den Geltungsverlust grundlegender Forschungsannahmen mit einer prinzipiellen Zurücknahme von wissenschaftlichen Erkenntnisansprüchen geantwortet.

Eine solche Reaktion kann schwerlich von der Enttäuschung über den stattfindenden Wahrheitsverlust frei sein. Es wundert deshalb nicht, daß Popper und Helmholtz an der Idee einer absoluten Wahrheit gemeinsam auf besondere Weise festhalten: Wahrheit bestimmt nicht nur das Ziel der Wissenschaft; sie soll auch nicht anders als wissenschaftlich, und d.h. für beide erfahrungswissenschaftlich, erreichbar sein. ${ }^{64}$ Bei Helmholtz verbindet sich dieser Szientismus noch mit der von ihm aufrechterhaltenen Orientierung an den Prinzipien der Mechanik. Auf die Krise seines Mechanismus antwortet er nicht mit einer Abkehr von dieser Naturauffassung. Im Gegenteil, mit dem Wandel seiner Wissenschaftsauffassung, die wissenschaftliche Erkenntnis über das Ganze der Natur hypothetisiert, kann er sie gegen Kritik immunisieren. ${ }^{65}$ Ähnliches ließe sich vermutlich auch über Poppers Szientismus sagen.

Wollte man beide Autoren in eine Entwicklungslinie empiristischer Wissenschaftstheorie einordnen, könnte man von einer fortschreitenden Distanzierung vom induktiven Verfahren sprechen, die mit einer zunehmenden Relativierung des Geltungsanspruches der wissenschaftlichen Erkenntnis einhergeht. An die Stelle eines uneingeschränkt behaupteten Geltungsanspruches der Induktion tritt zunächst seine Ergänzung um zusätzliche Verfahren der Bestätigung, die schließlich zusammen mit der Induktion verworfen werden. Darin spiegelt sich ein wachsender Skeptizismus gegenüber der Geltung der empirischen Basis. Ihr sind sowenig eindeutige Theorien zu entnehmen, wie sie ohne theoretische Vorannahmen zugänglich ist.

Helmholtz ist mit seiner lebenslangen Verpflichtung auf das induktive Verfahren dem modernen Element in Poppers Wissenschaftsauffassung nur sehr beschränkt entgegengekommen. Zu seinem Genie gehört aber, früh die Zeichen der Zeit gespürt zu haben. Er mag

63 Vgl. Schäfer (1988), 12 ff.

64 Mit der in seiner späteren Wissenschaftsauffassung vorgenommenen Relativierung des Gegensatzes von naturwissenschaftlicher und künstlerischer Erkenntnis revidiert Helmholtz nicht seine frühere Überzeugung, Naturerscheinungen ließen sich begrifflich nur durch das induktive Verfahren der Naturwissenschaft erfassen, sondern er präzisiert sie (vgl. Helmholtz (1862), $169 \mathrm{f}$, Helmholtz (1892), 338 ff.). Weitergehend kann man davon ausgehen, daß er zeitlebens in der Methode der Naturwissenschaft ein Vorbild für die Geisteswissenschaften gesehen hat (vgl. Cahan (1994)). Den Glauben, wissenschaftliche Probleme seien im Prinzip mit den Verfahren der Erfahrungswissenschaften lösbar, bezeichne ich mit dem Ausdruck „Szientismus“. Insbesondere im Hinblick auf seine Position zu den Sozialwissenschaften kann Poppers kritischer Rationalismus in diesem Sinn szientistisch genannt worden. Vgl. dazu Theodor W. Adorno, Theodor, Einleitung, in: Der Positivismusstreit in der deutschen Soziologie, Neuwied 1969, $12 \mathrm{ff}$.

65 Vgl. Schiemann (1994). 
geahnt haben, daß mit dem Fall absoluter Geltungsansprüche auch alle logischen Rekonstruktionen der Wissenschaft, wie sie Popper noch einmal versucht hat, zum Scheitern verurteilt sein könnten. Mathematik und Logik finden sich bei Helmholtz im Ansatz bereits der wahrnehmungstheoretisch begründeten Konzeption relativen Wissens untergeordnet. In ihr sucht er, das Geltungsproblem auch ohne Bezug auf die absoluten Instanzen einer realistisch angenommenen Wirklichkeit oder eines erfahrungsfreien Denkens zu lösen. Dieses Bemühen bringt ihn in die Nähe einer pragmatischen Wissenschaftskonzeption, die sich heute wachsender Zustimmung erfreut ${ }^{66}$ und deren Ablehnung Poppers kritische Distanz zur Moderne bezeichnet ${ }^{67}$. Ohne sich noch auf unhintergehbare reale oder geistige Geltungsgarantien zu stützen, sieht Helmholtz am Ende seiner Erkenntnistheorie „keine weitere Bürgschaft“ als den Erfolg einer jeden Tätigkeit und kann nicht mehr als „de(n) eine(n) Rath" geben: „Vertraue und handle!"68

Dipl.-Phys. Gregor Schiemann, Technische Hochschule Darmstadt, Institut für Philosophie, Residenzschloß, 64283 Darmstadt

\section{Literatur:}

Buchwald, Jed Z. (1994), Electrodynamics in Context: Object States, Laboratory Practice, and AntiRomanticism, in: Cahan (Hg.) (1994).

Cahan, David (1994), Helmholtz and the Civilizing Power of Science, in: ders. (Hg.) (1994).

Cahan, David (Hg.) (1994), Hermann von Helmholtz and the Foundations of Nineteenth-Century Science, Berkley u. a.

Conrat, Friedrich (1904), Hermann von Helmholtz' psychologische Anschauungen, Halle.

Diemer, Alwin (1968): Die Begründung des Wissenschaftscharakters der Wissenschaft im 19. Jahrhundert - Die Wissenschaftstheorie zwischen klassischer und moderner Konzeption, in: Alwin Diemer (Hg.), Beiträge zur Entwicklung der Wissenschaftstheorie im 19. Jahrhundert, Meisenheim am Glan.

Erdmann, Benno (1921), Die philosophischen Grundlagen von Hermann von Helmholtz' Wahrnehmungstheorie, Abh. Preuss. Akad., phil.-hist. Klasse, Berlin.

Feyerabend, Paul K. (1976), Wider den Methodenzwang, Frankfurt/M.

Galilei (1632), Galileo, Dialog über die beiden hauptsächlichen Weltsysteme, Darmstadt 1982.

- (1638), Galileo, Unterredungen und mathematische Demonstrationen über zwei neue Wissenszweige, die Mechanik und die Fallgesetze betreffend, Leipzig, Repr. Darmstadt 1964.

- (1890a ff.), Galileo, Le Opere, Edizione Nazionale, 22 Bde., Firenze.

Hatfield, Gary (1994), Helmholtz and Classicism: The Science of Aesthetics and the Aesthetics of Science, in: Cahan (Hg.) (1994)

Heidelberger, Michael (1994), Force, Law, and Experiment: The Evolution of Helmholtz's Philosophy of Science, in: Cahan (Hg.) (1994)

Heimann, P. M. (1974), Helmholtz and Kant: The Metaphysical Foundation of „Ueber die Erhaltung der Kraft"; in: Stud. Hist. Phil. Sci. 5, 205 ff.

Helmholtz, Hermann von (1847), Ueber die Erhaltung der Kraft, Leipzig 1889.

- (1853), Ueber Goethes naturwissenschaftliche Arbeiten, in: Helmholtz (1884), Bd. 1, 23 ff.

66 Stegmüller (1983 ff.), Bd. 1, 1 ff.

67 Popper (1973), 339 f., Popper (1956), 4 f., u.ö.

68 Helmholtz (1878a), 243 f., und entsprechend: Helmholtz (1892), 358. 
-(1854), Ueber die Wechselwirkung der Naturkräfte und die darauf bezüglichen neuesten Ermittelungen der Physik, in: Helmholtz (1884), Bd. 1, 48 ff.

- (1855), Ueber das Sehen des Menschen, in: Helmholtz (1884), Bd. 1, 85 ff.

- (1856 ff.), Handbuch der Physiologischen Optik, 1. Aufl., Leipzig 1956 ff.

- (1862), Ueber das Verhältniss der Naturwissenschaften zur Gesammtheit der Wissenschaften, in: Helmholtz (1884), Bd. 1, 157 ff.

- (1868a), Ueber die thatsächlichen Grundlagen der Geometrie, in: Helmholtz (1882 ff.), Bd. 2, 610 ff.

- (1868b), Ueber die Thatsachen, die der Geometrie zum Grunde liegen, in: Helmholtz (1882 ff.), Bd. $2,618 \mathrm{ff}$.

-(1869), Ueber das Ziel und die Fortschritte der Naturwissenschaft, in: Helmholtz(1884), Bd. 1, 157 ff.

- (1870), Ueber den Ursprung und die Bedeutung der geometrischen Axiome, in: Helmholtz (1884), Bd. 2, 1 ff.

- (1874a), ,Kritisches“. Vorwort zum zweiten Teil des ersten Bandes von W. Thomson und P.G. Tait, Handbuch der theoretischen Physik, autoris. dt. Übersetzung v. H. Helmholtz u. G. Wertheim, Braunschweig 1874. Unter dem Titel „Induction und Deduction“ auch enthalten in: Helmholtz (1884), Bd. 2, 413 ff. Ich zitiere nach dieser Ausgabe.

- (1874b), Vorwort zu John Tyndall: Fragmente aus den Naturwissenschaften, Braunschweig 1874. Unter dem Titel „Über das Streben nach Popularisirung der Wissenschaft“ auch enthalten in: Helmholtz (1884), Bd. 2, 422 ff. Ich zitiere nach dieser Ausgabe.

- (1878a), Die Thatsachen in der Wahrnehmung („Rektoratsrede“) mit drei Beilagen in: Helmholtz (1884), Bd. 2, 213 ff. (Rede) und 387 ff. (3 Beilagen); die 3. Beilage ist ein Auszug aus: Helmholtz (1878b) und wird nach diesem Text zitiert.

- (1878b), Ueber den Ursprung und Sinn der geometrischen Sätze. Antwort gegen Herrn Prof. Land, in: Helmholtz (1882 ff), Bd. 2, 640 ff.

- (1878c), Das Denken in der Medicin, 2., neu durchgearb. Aufl., Berlin. Auch enthalten in: Helmholtz (1884), Bd. 2, 165 ff. Ich zitiere nach dieser Ausgabe.

- (1881), Die neuere Entwickelung von Faraday's Ideen über Elektricität, in: Helmholtz (1884), Bd. 2, $249 \mathrm{ff}$.

- (1882 ff), Wissenschaftliche Abhandlungen, Leipzig, 3 Bde. (Bd.1: 1882, Bd.2: 1883, Bd.3: 1895).

- (1884), Vorträge und Reden, 2 Bde. Braunschweig, 5. Aufl. 1903.

- (1885 ff.), Handbuch der Physiologischen Optik, 2. umgearb. Aufl., Hamburg/Leipzig 1986.

- (1886), Antwortrede, gehalten beim Empfang der Graefe-Medaille, in: Helmholtz (1884), $311 \mathrm{ff}$.

- (1887), Rede über die Entdeckungsgeschichte des Princips der kleinsten Action, in: Adolf Harnack (Hg.), Geschichte der königl. preuss. Akademie der Wiss. zu Berlin, 3 Bde, Berlin 1900, Bd. 2, $282 \mathrm{ff}$.

- (1892), Goethe's Vorahnungen kommender naturwissenschaftlicher Ideen, in: Helmholtz (1884), Bd. 2, $335 \mathrm{ff}$.

-(1897 ff.), Vorlesungen über theoretische Physik, hg. v. Arthur König et al., 6 Bde., Leipzig.

Hempel, Carl G., und P. Oppenheim (1948), Studies in the Logic of Explanation, in: Philosophy of Science $15,98-115$.

Hörz, Herbert, und S. Wollgast (1971), Einleitung zu: Hermann von Helmholtz, Philosophische Vorträge und Aufsätze, Berlin.

Kaiser, Walter (1994): Helmholtz's Instrumental Role in the Formation of Classical Electrodynamics, in: Cahan (Hg.) (1994).

Kant, Immanuel (1787): Kritik der reinen Vernunft (2. Aufl.), in: Immanuel Kants gesammelte Schriften, hg. v. d. Königl. Preuß. (später: Dt.) Ak. d. Wiss. (zu Berlin), Berlin (1900 ff.).

Kirchhoff, Gustav (1876), Vorlesungen über Mechanik, Leipzig, 41897.

Koenigsberger, Leo (1902 f.): Hermann von Helmholtz, 3 Bde., Braunschweig.

König, Gert (1968), Der Wissenschaftsbegriff bei Helmholtz und Mach, in: Alwin Diemer (Hg.), Beiträge zur Entwicklung der Wissenschaftstheorie im 19. Jahrhundert, Meisenheim am Glan. 
Krüger, Lorenz (Hg.) (1994), Universalgenie Helmholtz, Berlin.

Kuhn, Thomas S. (1976), Die Struktur wissenschaftlicher Revolutionen, Frankfurt/M.

Mach, Ernst (1872), Die Geschichte und die Wurzel des Satzes von der Erhaltung der Arbeit, Prag.

Mill, John Stuart (1843), System der deduktiven und induktiven Logik, Teil 1 u. 2, in: ders., Ges.

Werke, Bd. 2 u. 3, Leipzig 1872.

Popper, Karl R. (1930 ff.), Die beiden Grundprobleme der Erkenntnistheorie, Tübingen 1979.

- (1935), Die Logik der Forschung, Tübingen ${ }^{9} 1989$.

- (1956), Postscript to the Logic of Scientific Discovery, vol. 2 (The Open Universe) u. vol. 3 (Quantum Theory and the Schism in Physics), London u. a. 1982.

- (1963), Conjectures and Refutation. The Growth of Scientific Knowledge, London.

- (1973), Objektive Erkenntnis, Hamburg.

Pulte, Helmut (1993), Zum Niedergang des Euklidianismus in der Mechanik des 19. Jahrhunderts, In:

Neue Realitäten. XVI. Dt. Kongreß für Philosophie, Berlin 1994, 833 ff.

Rechenberg, Helmut (1994), Hermann von Helmholtz, Weinheim/New York.

Riemann, Bernhard (1867), Über die Hypothesen, welche der Geometrie zugrunde liegen, Darmstadt 1959.

Rorty, Richard (1981), Der Spiegel der Natur, Frankfurt/M.

Schäfer, Lothar (1988), Karl R. Popper, München

Schiemann, Gregor (1994), Die Hypothetisierung des Mechanismus, in: Krüger (Hg.) (1994).

Schilpp, P.A. (Hg.) (1974), The Philosophy of Karl Popper, 2 Bde, La Salle (Ill.).

Schnädelbach, Herbert (1983), Philosophie in Deutschland 1831 - 1933, Frankfurt/M.

Stegmüller, Wolfgang (1983 ff.), Probleme und Resultate der Wissenschaftstheorie und der Analytischen Philosophie, Berlin u. a.

Turner, R. Steven (1994), Consensus and Controversy: Helmholtz and the Visual Perception of Space, in: Cahan (Hg.) (1994).

Welsch, Wolfgang (1988), Unsere postmoderne Moderne, 2. Aufl., Weinheim.

Winters, Stephen M. (1985), Hermann von Helmholtz's Discovery of Force Conservation, Diss. Baltimore. 


\section{DEUTSCHE ZEITSCHRIFT FÜR $P H\|L O S O P H\| E$}

Zweimonatsschrift der internationalen philosophischen Forschung

\section{Sonderdruck}

$5 / 1995$

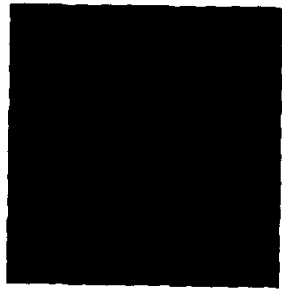

Nicholas Rescher Philosophie am Ende des Jahrhunderts Renate Wahsner Die Newtonsche Vernunft und ihre Hegelsche Kritik Cornelia Klinger Theorie der Geschlechterdifferenz Hans-Ulrich Lessing Dilthey und Helmholtz Michael Heidelberger Helmholtz als Philosoph Gregor Schiemann Zwischen klassischer und moderner Wissenschaftstheorie Bertolt Fessen Mißlungene Verständigung - Philosophen zum neven Deutschland Giuseppe Cacciatore Gianbattista Vico Marco Bonato Rehabilitierung der praktischen Utopie 